\title{
Model-Corrected Microwave Imaging through Periodic Wall Structures
}

\author{
Paul C. Chang, ${ }^{1}$ Robert J. Burkholder, ${ }^{2}$ and John L. Volakis ${ }^{2}$ \\ ${ }^{1}$ Science Applications International Corporation, 4031 Colonel Glenn Highway, Beavercreek, OH 45431, USA \\ ${ }^{2}$ Department of Electrical and Computer Engineering, Ohio State University, 2015 Neil Avenue, Columbus, OH 43210, USA
}

Correspondence should be addressed to Paul C. Chang, paul.chang.osu@gmail.com

Received 2 February 2012; Accepted 19 March 2012

Academic Editor: Danilo Erricolo

Copyright (C) 2012 Paul C. Chang et al. This is an open access article distributed under the Creative Commons Attribution License, which permits unrestricted use, distribution, and reproduction in any medium, provided the original work is properly cited.

\begin{abstract}
A model-based imaging framework is applied to correct the target distortion seen in microwave imaging through a periodic wall structure. In addition to propagation delays caused by the wall, it is shown that the structural periodicity induces high-order space harmonics leading to other ghost artifacts in the through-wall image. To overcome these distortions, the periodic layer Green's function is incorporated into the forward model. A linear back-projection solution and a nonlinear minimization solution are applied to solve the inverse problem. The model-based back-projection image corrects the distortion and has higher resolution compared with free space due to the inclusion of multipath propagation through the periodic wall, but considerable sidelobe clutter is present. The nonlinear solution not only corrects target distortion without clutter but also reduces the solution to a sparse form.
\end{abstract}

\section{Introduction}

Electromagnetic sensing has been routinely used for imaging selected objects in obstructive complex environments. Among them, ground-penetrating radars (GPR), operating in UHF/VHF frequencies, have been used to detect landmines and locate buried targets $[1,2]$. Synthetic aperture radars (SARs) have also been extensively used for land surface and subsurface mapping. As is well known, all such RF sensing systems suffer from multiscattering effects that distort the image. This issue is even more pronounced in through-wall radar imaging [3-5] where the wall can cause image dislocation, deformation, and ghosting due to propagation delay, ray bending, and higher order modal scattering from structural periodicity [6].

Several efforts have been published on improving through-wall imaging [7-11]. However, these works have primarily focused on imaging through homogeneous layered walls. In this paper, we instead focus on an imaging framework aimed at mitigating multi-scattering from periodic walls. Specifically, we introduce a corrective approach that overcomes issues with RF propagation through a periodic wall. When compared to our previous work in [12-14], this paper provides a more generalized framework that can be used to correct propagation distortions and enhance target resolution. Rather than using numerical techniques to characterize propagation through periodic structures [1518], a much faster near zone Floquet modal solution [19, 20] is incorporated into a linear forward model. A backprojection (BP-) based image is then obtained from the forward model using a diagonal approximation to the leastsquares matrix solution. Due to the multipath introduced by the Floquet modes, the BP solution actually gives a higher resolution image than in free space. However, it also introduces high side-lobes and clutter into the image.

To overcome the limitations of the BP solution, Cetin and Karl's nonlinear minimization algorithm [21] is applied to attain a sparse solution of the forward model that superresolves the image while also avoiding the side-lobes and clutter of the linear solution. An efficient translationinvariant point spread function (PSF) approximation is introduced to greatly reduce the computational cost of the non-linear minimization algorithm.

Below, we begin (Section 2) by formulating the forward model that characterizes the scattering from an ideal point scatterer behind a periodic wall. Next, Section 3 describes 


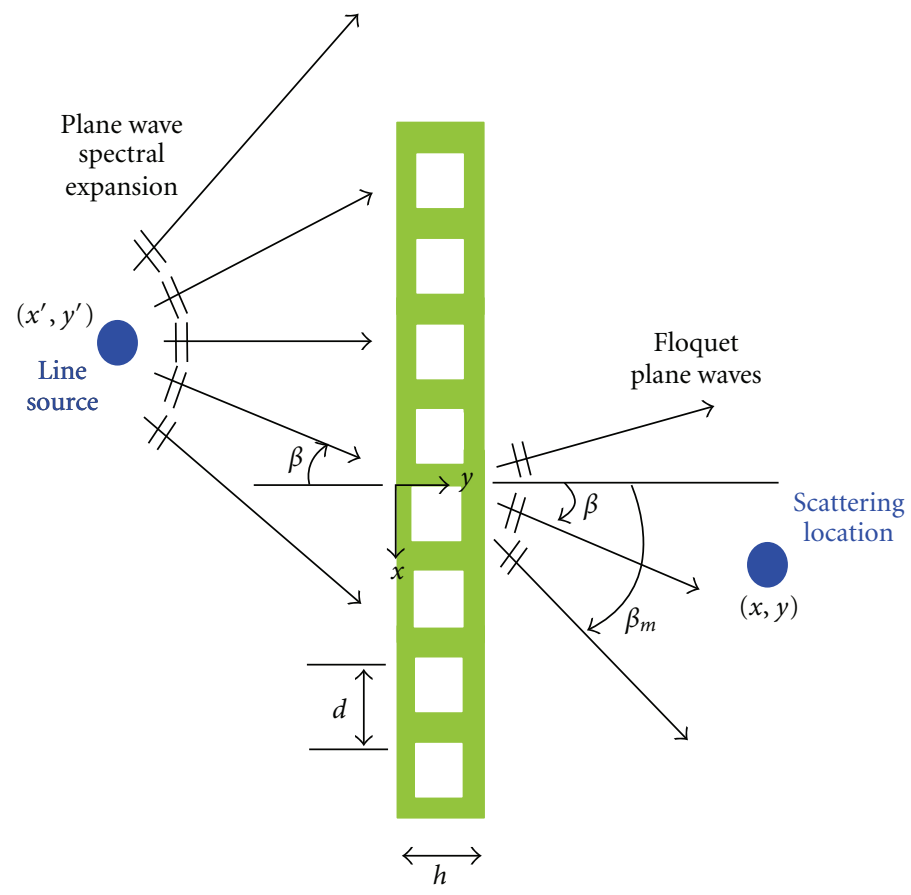

FIGURE 1: Line source illumination of a periodic wall. Radiated fields from the line source are represented with a plane wave spectral expansion. Each plane wave gives rise to another set of transmitted Floquet plane waves.

the proposed imaging methods that incorporate the forward model to restore the image of targets behind the wall. Simulated imaging results are presented in Section 4. The formulation is presented for a two-dimensional (2D) problem and may be extended to $3 \mathrm{D}$ in a straightforward manner. An $e^{j \omega t}$ harmonic time convention is assumed and suppressed.

\section{Forward Model for the Near-Zone Scattering from Targets Seen through a Periodic Wall}

Let us consider the near field illumination of a periodic wall as depicted in Figure 1. The spectral form of the incident field radiated by a unit amplitude line source is given in [19] as:

$$
\begin{aligned}
E_{z}(\bar{\rho}) & =-\frac{k_{0} Z_{0}}{4} H_{0}^{(2)}\left(k_{0}\left|\bar{\rho}-\bar{\rho}^{\prime}\right|\right) \\
& =-\frac{k_{0} Z_{0}}{4 \pi} \int_{-\pi / 2}^{\pi / 2} e^{-j k_{0} \cdot\left(y-y^{\prime}\right) \cos \beta-j k_{0}\left(x-x^{\prime}\right) \sin \beta} \cos \beta d \beta,
\end{aligned}
$$

where $\bar{\rho}^{\prime}=\left(x^{\prime}, y^{\prime}\right)$ and $\bar{\rho}=(x, y)$ refer to the line source and receiver locations. $\bar{k}=\hat{x} k_{x}+\hat{y} k_{y}$ with $\beta$ denoting the incident angle measured from the $y$ axis. $Z_{0}$ and $k_{0}$ are the free space impedance and wave number, respectively, and $H_{0}^{(2)}$ is the zero-order Hankel function of the second kind. As the integral in (1) can be interpreted as a summation of plane waves, each plane wave then gives rise to a discrete set of transmitted Floquet modes (see Figure 1). The transmission through the periodic wall takes the form:

$$
\begin{aligned}
E_{z}(\bar{\rho})= & -\frac{k_{0} Z_{0}}{4 \pi} \int_{-\pi / 2}^{\pi / 2} e^{j k_{0} x^{\prime} \sin \beta+j k_{0} y^{\prime} \cos \beta} \\
& \cdot\left[\sum_{m=-\infty}^{\infty} T_{m}(\beta) \cdot e^{-j k_{0} x \sin \beta_{m}-k_{0}(y-h) \cos \beta_{m}}\right] \cos \beta d \beta .
\end{aligned}
$$

Here, $T_{m}$ and $\beta_{m}$ are the transmission coefficients and associated propagation angles, respectively, of the Floquet modes generated by the plane wave $e^{j k_{0}(x \cos \beta+y \sin \beta)}$. The outgoing direction of the Floquet modes is defined by:

$$
k_{0} \sin \beta_{m}=k_{0} \sin \beta+\frac{2 \pi m}{d} \quad m=0, \pm 1, \pm 2, \pm 3 \cdots
$$

with $d$ being the unit cell length along the $x$ direction. As usual, $m=0$ refers to the directly transmitted mode satisfying Snell's law. For $m \neq 0$, the outgoing plane waves only propagate when $\left|\sin \beta_{m}\right| \leq 1$ (that is, they are evanescent otherwise). All propagating modes are used in the results that follow, and no evanescent modes are used. Inclusion of evanescent modes is not needed since the imaging points are far away from the wall. The associated transmission coefficients in (2) are computed via the mode matching solution of $[19,20]$.

A scattering problem can typically be formulated as the linear superposition of the first-order scattering from a set of ideal point scatterers, assuming no interactions between the 


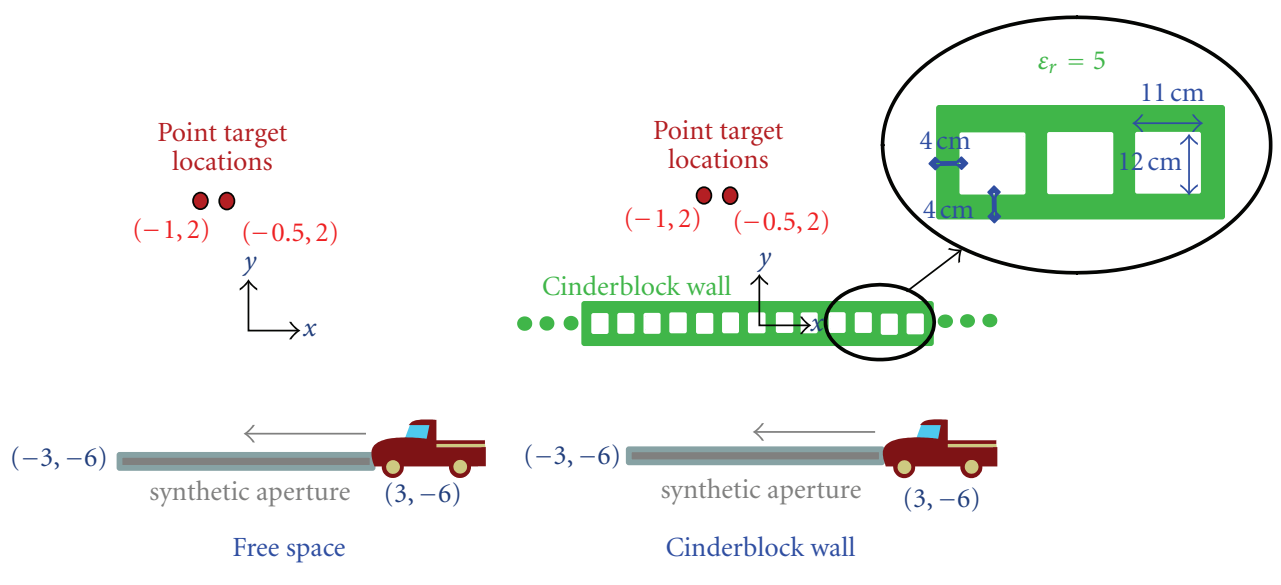

(a)

(b)

FIGURE 2: Illustration of the near zone through-wall sensing: (a) in the absence of a wall, (b) in the presence of a periodic cinderblock wall.

points. This leads to the definition of a discrete forward model given by:

$$
\mathbf{s}=\text { Af }+\mathbf{n},
$$

where $\mathbf{s}$ is a column vector of length $Q$ and contains the amplitude and phase of the scattered fields, and $\mathbf{f}$ is a column vector of length $N$ representing the reflectivity of the imaged points (pixels). Consequently, $\mathbf{A}$ is the $Q \times N$ forward model matrix describing the two-way propagation between the sensors and reflecting pixels. Finally, $\mathbf{n}$ is a residual vector that contains noise, extraneous scattering (clutter), and model mismatch errors. The elements of $\mathbf{A}$ are found from (2). For example, for monostatic sensing, the elements are given by,

$$
A_{q n}=\left.\left[E_{z}\left(\bar{\rho}_{n}\right)\right]^{2}\right|_{\bar{\rho}^{\prime}=\bar{\rho}_{q}^{\prime}},
$$

where $\bar{\rho}_{n}$ is the location of the $n$th pixel and $\bar{\rho}_{q}^{\prime}$ is the source location of the $q$ th measurement. It is noted that the index $q$ also encompasses multiple frequencies ( $k_{0}$ values) in the phase history domain. The square of $E_{z}$ in (5) follows from the reciprocity relationship between the paths from the line source to the scattering point and back. The linear forward model of (4) may now be solved as an inverse problem as described in the next section.

\section{Inverse Imaging Solutions}

In generating an image of the scatterers behind the wall, we must solve for $\mathbf{f}$ using the received scattering vector $\mathbf{s}$ and $\mathrm{a}$ good approximation of the matrix A. Since $Q$ is in general not equal to $N$, the least squares solution of (4) may be formulated as:

$$
\mathbf{f}=\left[\mathbf{A}^{H} \mathbf{A}\right]^{-1} \mathbf{A}^{H} \mathbf{s}
$$

where the superscript " $H$ " denotes the complex conjugate transpose (or Hermitian) of matrix A. Typically, the image space is more densely sampled than the intrinsic resolution of the imaging system, which results in the pixels having linear dependencies and the matrix $\mathbf{A}^{H} \mathbf{A}$ becoming rankdeficient. In that case, (6) cannot be solved directly without the use of regularization or a pseudoinverse. However, it is expected that $\mathbf{A}^{H} \mathbf{A}$ is diagonally dominant even if the pixels are somewhat linearly dependent. A fast solution may, therefore, be found by approximating $\mathbf{A}^{H} \mathbf{A}$ with its diagonal elements yielding:

$$
\mathbf{f} \approx\left(\operatorname{diag}\left[\mathbf{A}^{H} \mathbf{A}\right]\right)^{-1} \mathbf{A}^{H} \mathbf{s}
$$

We observe that (7) is evocative of the well-known "SAR back-projection" or "conjugate-phase matched filter" imaging methods. Indeed, (7) is a diagonally weighted generalization of these concepts. Herewith, we will refer to (7) as the back-projection (BP) method. The BP image may be generated for the cost of a single matrix-vector product.

As stated above, when the image space is oversampled, the pixels become linearly dependent. Hence there are an infinite number of solutions that satisfy (4). We may, therefore, apply some criterion to choose the most optimal solution based on the assumed scattering model. For higher resolution, we choose the solution that enhances the "sparseness." This may be posed mathematically as finding a solution $\tilde{\mathbf{f}}$, which satisfies

$$
\widetilde{\mathbf{f}}=\min \|\mathbf{f}\|_{p} \quad \text { subject to }\|\mathbf{s}-\mathbf{A f}\|_{2} \leq \varepsilon .
$$

Here $\|\mathbf{f}\|_{p}$ denotes the $p$-norm of the vector $\mathbf{f}$ defined in [21] as

$$
\|\mathbf{f}\|_{p} \approx \sum_{i=1}^{N}\left(\left|\mathbf{f}_{i}\right|^{2}+\delta\right)^{p / 2},
$$

where $\delta$ is a small positive constant, $N$ is the length of complex vector $\mathbf{f}$, and $\mathbf{f}_{i}$ is the $i$ th element of $\mathbf{f}$. The norm is defined for any real number $p \geq 0$, and when $0 \leq p \leq$ 1 , (8) obtains the sparse solution and enhances the target resolution by minimizing the number of nonzero pixels in the image [21]. 


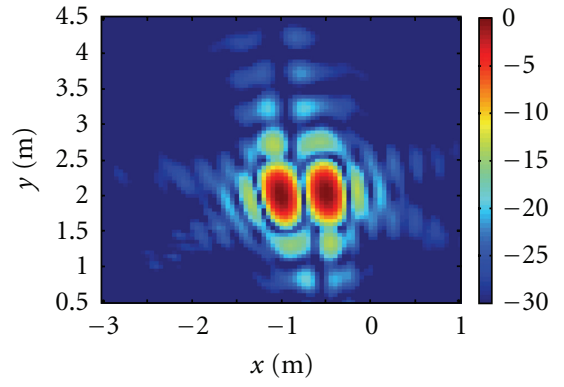

(a)

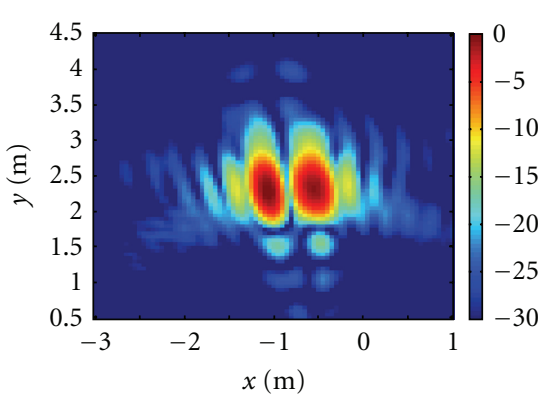

(b)

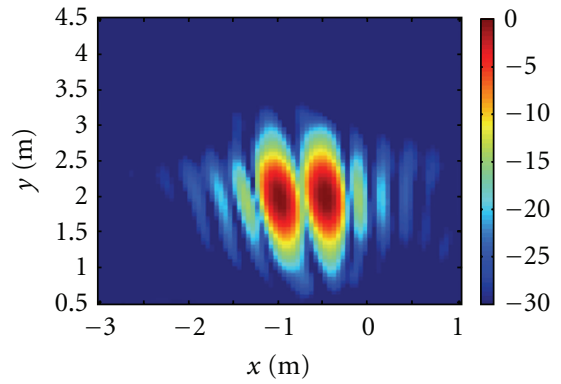

(c)

FIGURE 3: Back-projection images of the two point targets for the configuration in Figure 2 with data collected over the band 0.7-1.0 GHz: (a) image in free space, (b) Image in presence of the cinderblock wall using the free space Green's function, (c) image in presence of the cinderblock wall using the periodic layer Green's function.

It is possible to define an optimization function based directly on (8), but for reasons that will become clear we again use the least-squares form of (4), or the image domain forward model,

$$
\mathbf{A}^{H} \mathbf{s}=\mathbf{A}^{H} \mathbf{A f}+\mathbf{A}^{H} \mathbf{n}
$$

or equivalently,

$$
\mathbf{g}=\mathbf{B f}+\mathbf{n}_{\text {id }},
$$

where $\mathbf{g}=\mathbf{A}^{H} \mathbf{s}, \mathbf{B}=\mathbf{A}^{H} \mathbf{A}$, and $\mathbf{n}_{\mathrm{id}}$ is the noise/clutter vector in the image domain. $\mathbf{B}$ is an $N \times N$ matrix whose columns may be interpreted as the point spread functions (PSFs) of the pixels and transforms the ideal image $f$ to a degraded image $\mathbf{g}$, which is simply the unweighted BP solution. In practice, it is advantageous to formulate the forward model this way so that the size of the $\mathbf{B}$ matrix does not depend on the measured data and provides more flexibility in focusing in on a particular region of the image domain. A computational advantage of this formulation is that the PSFs (columns of $\mathbf{B}$ ) are approximately translationinvariant, so only one PSF needs to be generated and stored. Otherwise, B could be an intractably large matrix to deal with.

As suggested by [21], the sparse solution of (8) may be obtained by minimizing the functional for $0 \leq p \leq 1$,

$$
\psi(\mathbf{f})=\|\mathbf{g}-\mathbf{B f}\|_{2}+\lambda\|\mathbf{f}\|_{p},
$$

where $\lambda$ is a positive real parameter chosen $a$ priori to adjust the relative weight of the sparseness versus the error in the forward model. Physically, this penalization term serves to enhance the point-based features in the reconstruction, and is self-consistent with the point-target assumption of the forward model. We shall refer to (12) as the "sparse imaging" approach. Interested readers may also see [22] with regards to the choice of regularization parameters. Here, (12) is minimized using the quasi-Newton iterative algorithm with modified Hessian update as described in [21]. It is noted that there are more efficient algorithms available for obtaining a sparse point-target representation of the image (e.g., matching and basis pursuits, iterative thresholding, etc.).
However, these methods generally start with the null solution and add points until the forward model is satisfied. These points may not necessarily correspond to true scattering centers and may not, therefore, super-resolve the image. This is in contrast to the gradient-type optimization used here, which starts with the BP solution and iteratively sharpens the image.

\section{Simulated Imaging Results}

To demonstrate the imaging approaches described in the previous section, let us consider the through-wall sensing example in Figure 2. Specifically, two point targets are placed at $(x, y)=(-1 \mathrm{~m}, 2 \mathrm{~m})$ and $(x, y)=(-0.5 \mathrm{~m}, 2 \mathrm{~m})$. The monostatic radar is located at $y=-6 \mathrm{~m}$ and was moved from $x=-3$ to $3 \mathrm{~m}$ (in $0.05 \mathrm{~m}$ step) to obtain the vector $\mathbf{s}$ in the frequency ranges $0.7-1.0 \mathrm{GHz}$ and $1.7-2.0 \mathrm{GHz}$. Only the $m=0$ Floquet mode is present in the lower frequency band, whereas the $m=-1,0,1$ modes are present in the upper frequency band. The measurements were corrupted by random white noise with $15 \mathrm{~dB}$ signal-to-noise ratio (SNR). The A matrix is constructed for two cases: (a) in the absence of a wall (free space) and (b) in the presence of a periodic cinderblock wall. The wall is assumed infinite in the $x$ and $z$ directions. In the following, all of the images are normalized to $0 \mathrm{~dB}$ peak magnitude.

The back-projection images computed from (7) for the two different frequency bands are depicted in Figures 3 and 4, respectively. The (a) images are in free space, and the (b) images are in the presence of the cinderblock wall using the free space Green's function. It is evident that the lower frequency images exhibit lower cross-range resolution, as expected. However, they are less susceptible to wall distortions. It is also seen that the walls introduce propagation delays leading to shifted and distorted targets. In the higher frequency band, the higher-order Floquet modes cause drastic distortion and ghosting as seen in Figure 4(b).

To compensate the wall effects, the back-projection method was reapplied with $\mathbf{A}$ incorporating the appropriate wall Green's function. The corresponding images are shown in Figures 3(c) and 4(c). These images display the true locations of the targets, although the shape is not completely 


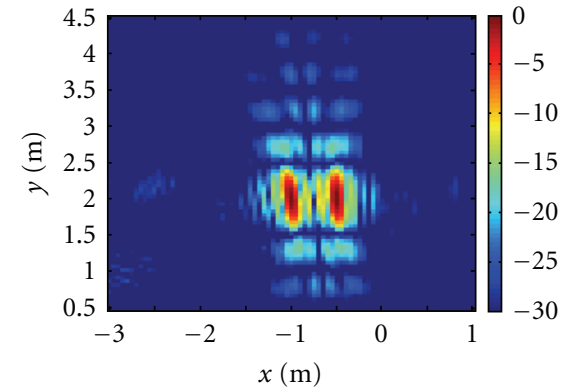

(a)

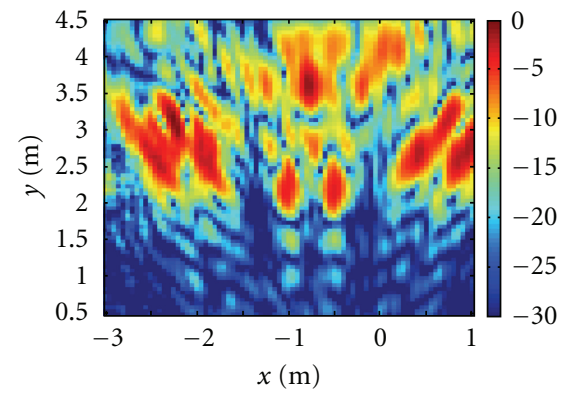

(b)

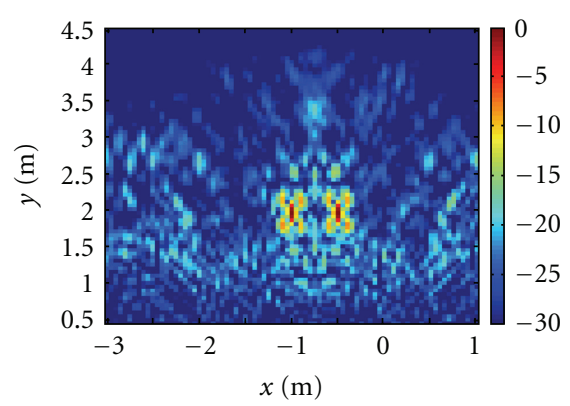

(c)

FIGURE 4: Back-projection images of the two point targets for the configuration in Figure 2 with data collected over the band 1.7-2.0 GHz: (a) Image in free space, (b) image in presence of the cinderblock wall using the free space Green's function, (c) image in presence of the cinderblock wall using the periodic layer Green's function.

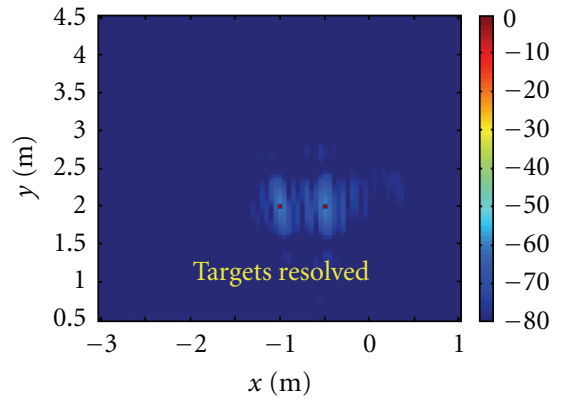

(a)

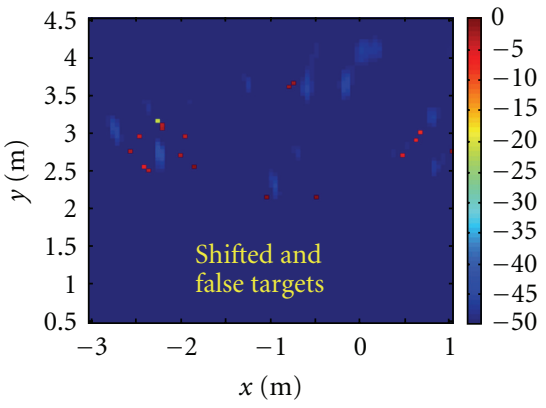

(b)

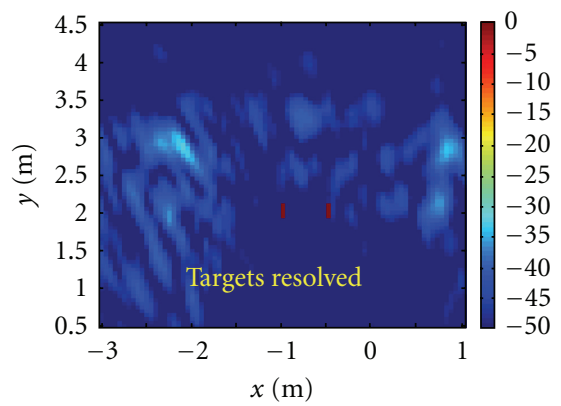

(c)

Figure 5: Sparse images ( $P=0.8, \lambda=9,20$ iterations) of the two point targets for the configuration in Figure 2 with data collected over the band 1.7-2.0 GHz: (a) image in free space, (b) image in presence of the cinderblock wall using the free space Green's function, (c) image in presence of the cinderblock wall using the periodic layer Green's function.

restored with respect to the free space image. This is due to the fact that some frequencies will inevitably be filtered out by the wall. A very interesting phenomenon is seen in Figure 4(c), where the image appears to be more finely resolved than the free space image of Figure 4(a). Such "super-focusing" is due to exploiting the multipath associated with the Floquet modes. (It is noted that no evanescent modes are used in this analysis, so the superfocusing cannot be attributed to evanescent effects as often claimed in other near-field imaging works.) In the same way as multipath propagation expands the information capacity of a communication channel, multiple reflections can lead to improved image resolution $[23,24]$.

The latter serves to increase the effective aperture, but at the expense of introducing more imaging sidelobes.

Next, the sparse images computed by minimizing (12) are presented in Figure 5 for the $1.7-2.0 \mathrm{GHz}$ frequency band. As seen in Figure 5(a), in free space the two point targets are resolved to the size of individual pixels and the sidelobes are very low on an $80 \mathrm{~dB}$ color scale. Figure 5(b) shows the sparse through-wall image generated using the free space Green's function. The sidelobes have been suppressed, and the point targets are more clearly seen, but they appear shifted from their true locations and there are several false targets. The cinderblock wall image is restored in Figure 5(c) using the appropriate periodic layer Green's function, and the sidelobes are reduced to $-30 \mathrm{~dB}$ relative to the peaks.

Back-projection and sparse imaging methods are also applied to image a metallic plate with the same scanning setup as in Figure 2 for frequency range $1.7-2.0 \mathrm{GHz}$. The plate extends horizontally from $x=-1$ to $-0.5 \mathrm{~m}$ at the vertical distance of $y=2 \mathrm{~m}$. The output images are displayed in Figures 6 and 7, respectively. Once again Floquet multipath propagations severely degrade the back-projection image (see Figure 6(b)), but if they are compensated for during the imaging process, we may instead use them to our advantage for resolution enhancement (at the cost of more imaging sidelobes) as shown in Figure 6(c). Sparse imaging, on the other hand, provides much sharper image outputs. If the wall has been accounted for via appropriate periodic layer Green's function, most of the false alarms due to Floquet multi-paths can be removed.

We remark that a translation-invariant PSF approximation is used to generate the sparse images of Figures 5 and 7 as discussed in Section 3. Specifically in this example, a single PSF for a point located at the center pixel of the grid is first computed. The PSFs for all other pixels are then computed approximately via $2 \mathrm{D}$ convolution. The more exact spatially varying PSFs may be adapted to further improve image quality but at a greatly increased computational cost. 


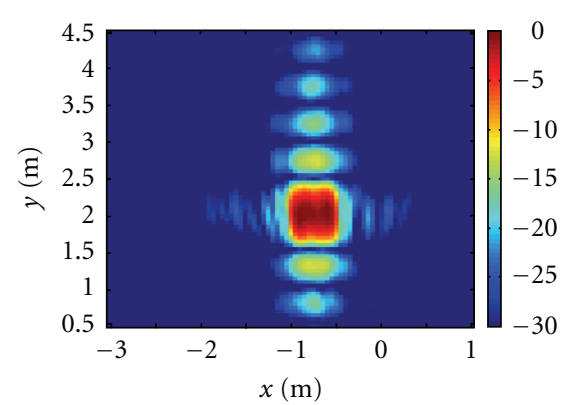

(a)

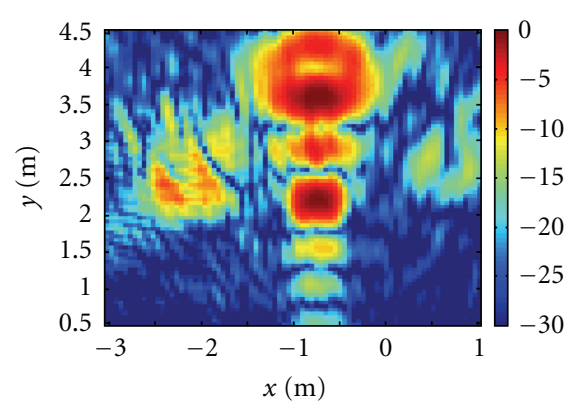

(b)

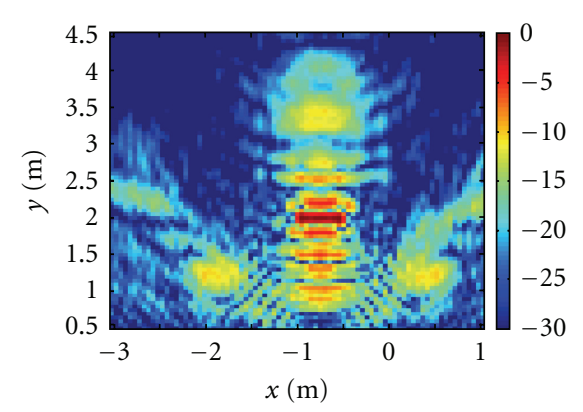

(c)

FIGURE 6: Back-projection images of a metallic plate extending from $x=-1$ to -0.5 at $y=-2$ for the configuration in Figure 2 with data collected over the band 1.7-2.0 GHz: (a) image in free space, (b) image in presence of the cinderblock wall using the free space Green's function, (c) image in presence of the cinderblock wall using the periodic layer Green's function.

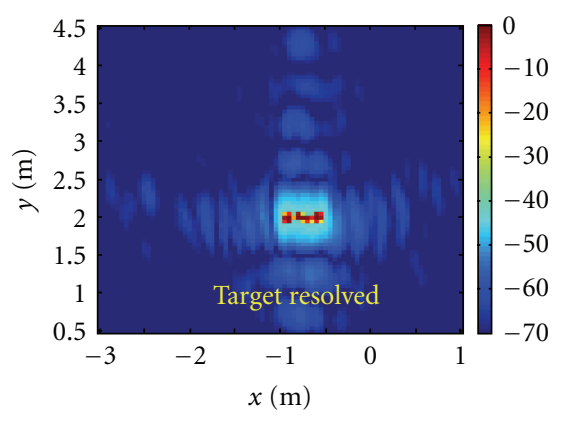

(a)

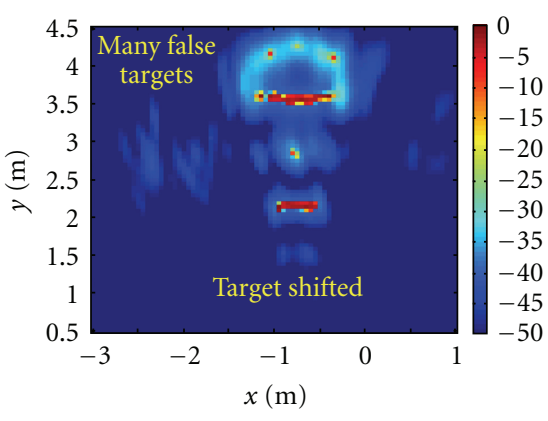

(b)

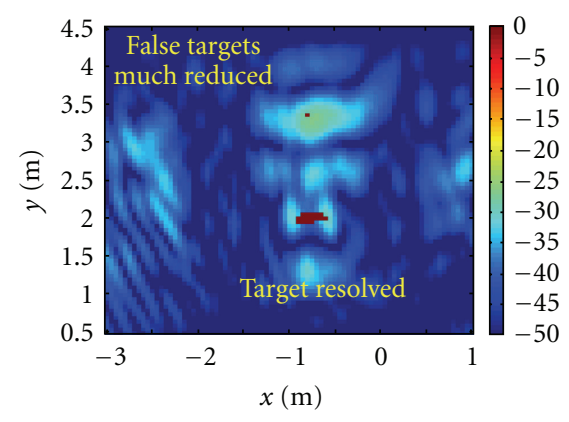

(c)

Figure 7: Sparse images ( $P=0.8, \lambda=9,20$ iterations) of a metallic plate extending from $x=-1$ to -0.5 at $y=-2$ for the configuration in Figure 2 with data collected over the band 1.7-2.0 GHz: (a) image in free space, (b) image in presence of the cinderblock wall using the free space Green's function, (c) image in presence of the cinderblock wall using the periodic layer Green's function.

Computationally, construct of the central PSF and the initial back-projection image from the forward model and its Hermitian adjoint are the most time-consuming steps. For the considered numerical example (with 101 frequency points, 121 sensor locations, and $81 \times 81$ imaging pixels), it took approximately 12 minutes for the back-projection to generate a model-corrected image on a standard laptop CPU (Intel(R) Core(TM) 2 Duo T7300 at $2.00 \mathrm{GHz}$ ) and 15 minutes for the sparse imaging algorithm. That is, even though the sparse imaging takes many iterations, it is very fast because most of the time is spent generating the central PSF and initial back-projection image.

\section{Conclusion}

Imaging techniques based on a forward model framework have been carried out to restore interior targets for nearzone through-wall surveillance. Specifically, they can be used to compensate the propagation delays and Floquet multiscattering of a periodic wall while enhancing the target resolution. The Floquet multipaths were shown to superresolve targets in the back-projection image (without inclusion of evanescent modes), but significant sidelobe clutter was introduced. The sparse imaging method is able to superresolve targets directly while correcting for target distortion. However, if the correct periodic wall model is not incorporated, the targets are displaced and several false alarms appear as well. These techniques can also be applicable to more general surveillance problems involving different medium Green's functions.

In this paper we used a known periodic wall model with a known orientation relative to the source. In the future, it is of interest to allow uncertainty in the parameters of the wall model. For example, the periodicity, thickness, dielectric constant, and relative orientation may be left as variables in the optimization process. The model-based approach may then be applied to measured or numerically generated data.

\section{References}

[1] D. J. Daniels, Ed., Ground Penetrating Radar, IEEE, 2004.

[2] H. M. Jol, Ed., Ground Penetrating Radar: Theory and Applications, Elsevier B.V., 2009.

[3] J. Richards, Remote Sensing With Imaging Radar (Signals and Communication Technology), Springer, 2009.

[4] U. Soergel, Radar Remote Sensing of Urban Areas (Remote Sensing and Digital Image Processing), Springer, 2010.

[5] E. J. Baranoski, "Through wall imaging: historical perspective and future directions," in Proceedings of the IEEE International Conference on Acoustics, Speech and Signal Processing (ICASSP '08), pp. 5173-5176, Las Vegas, Nev, USA, April 2008. 
[6] H. L. Bertoni, L. H. S. Cheo, and T. Tamir, "Frequencyselective reflection and transmission by a periodic dielectric layer," IEEE Transactions on Antennas and Propagation, vol. 37, no. 1, pp. 78-83, 1989.

[7] M. Dehmollaian and K. Sarabandi, "Refocusing through building walls using synthetic aperture radar," in Proceedings of the IEEE International Symposium on Antenna and Propagation, pp. 1465-1468, June 2007.

[8] F. Ahmad, M. G. Amin, and S. A. Kassam, "Synthetic aperture beamformer for imaging through a dielectric wall," IEEE Transactions on Aerospace and Electronic Systems, vol. 41, no. 1, pp. 271-283, 2005.

[9] P. C. Chang, R. J. Burkholder, and J. L. Volakis, "Adaptive CLEAN with target refocusing for through-wall image improvement," IEEE Transactions on Antennas and Propagation, vol. 58, no. 1, pp. 155-162, 2010.

[10] R. Solimene, F. Soldovieri, G. Prisco, and R. Pierri, "Threedimensional through-wall imaging under ambiguous wall parameters," IEEE Transactions on Geoscience and Remote Sensing, vol. 47, no. 5, pp. 1310-1317, 2009.

[11] R. Solimene, R. Di Napoli, F. Soldovieri, and R. Pierri, “TWI for an unknown symmetric lossless wall," IEEE Transactions on Geoscience and Remote Sensing, vol. 49, no. 8, pp. 2876-2886, 2011.

[12] R. J. Burkholder and J. L. Volakis, "Time-reversal radar imaging through periodic structures," in Proceedings of the IEEE International Symposium on Antennas and Propagation and USNC/URSI National Radio Science Meeting, pp. 1-4, North Charleston, SC, USA, 2009.

[13] R. J. Burkholder, "Electromagnetic models for exploiting multi-path propagation in through-wall radar imaging," in Proceedings of the International Conference on Electromagnetics in Advanced Applications (ICEAA '09), pp. 572-575, Torino, Italy, September 2009.

[14] P. C. Chang, R. J. Burkholder, and J. L. Volakis, "Time-reversal processing and autofocus of targets behind complex wall," in Proceedings of the IEEE International Symposium on Antennas and Propagation, pp. 1-4, Toronto, Canda, July 2010.

[15] R. A. Dalke, C. L. Holloway, P. McKenna, M. Johansson, and A. S. Ali, "Effects of reinforced concrete structures on RF communications," IEEE Transactions on Electromagnetic Compatibility, vol. 42, no. 4, pp. 486-496, 2000.

[16] C. F. Yang, W. D. Burnside, and R. C. Rudduck, "A periodic moment method solution for TM scattering from lossy dielectric bodies with application to wedge absorber," IEEE Transactions on Antennas and Propagation, vol. 40, no. 6, pp. 652-660, 1992.

[17] T. F. Eibert, J. L. Volakis, D. R. Wilton, and D. R. Jackson, "Hybrid FE/BI modeling of 3-D doubly periodic structures utilizing triangular prismatic elements and an MPIE formulation accelerated by the Ewald transformation," IEEE Transactions on Antennas and Propagation, vol. 47, no. 5, pp. 843-850, 1999.

[18] M. Dehmollaian and K. Sarabandi, "Simulation of throughwall microwave imaging: forward and inverse models," in Proceedings of the IEEE International Geoscience and Remote Sensing Symposium (IGARSS '06), pp. 407-410, Denver, Colo, USA, August 2006.

[19] W. Honcharenko and H. L. Bertoni, "Transmission and reflection characteristics at concrete block walls in the UHF bands proposed for future PCS," IEEE Transactions on Antennas and Propagation, vol. 42, no. 2, pp. 232-239, 1994.
[20] S. V. Savov and M. H. A. J. Herben, "Modal transmissionline modeling of propagation of plane radiowaves through multilayer periodic building structures," IEEE Transactions on Antennas and Propagation, vol. 51, no. 9, pp. 2244-2251, 2003.

[21] M. Çetin and W. C. Karl, "Feature-enhanced synthetic aperture radar image formation based on nonquadratic regularization," IEEE Transactions on Image Processing, vol. 10, no. 4, pp. 623-631, 2001.

[22] F. Soldovieri, R. Solimene, L. Lo Monte, M. Bavusi, and A. Loperte, "Sparse reconstruction from GPR data with applications to rebar detection," IEEE Transactions on Instrumentation and Measurement, vol. 60, no. 3, pp. 1070-1079, 2011.

[23] D. Liu, G. Kang, L. Li et al., "Electromagnetic time-reversal imaging of a target in a cluttered environment," IEEE Transactions on Antennas and Propagation, vol. 53, no. 9, pp. 30583066, 2005.

[24] R. Solimene, R. Barresi, and G. Leone, "Localizing a buried planar perfect electric conducting interface by multi-view data," Journal of Optics A, vol. 10, no. 1, Article ID 015010, 2008. 

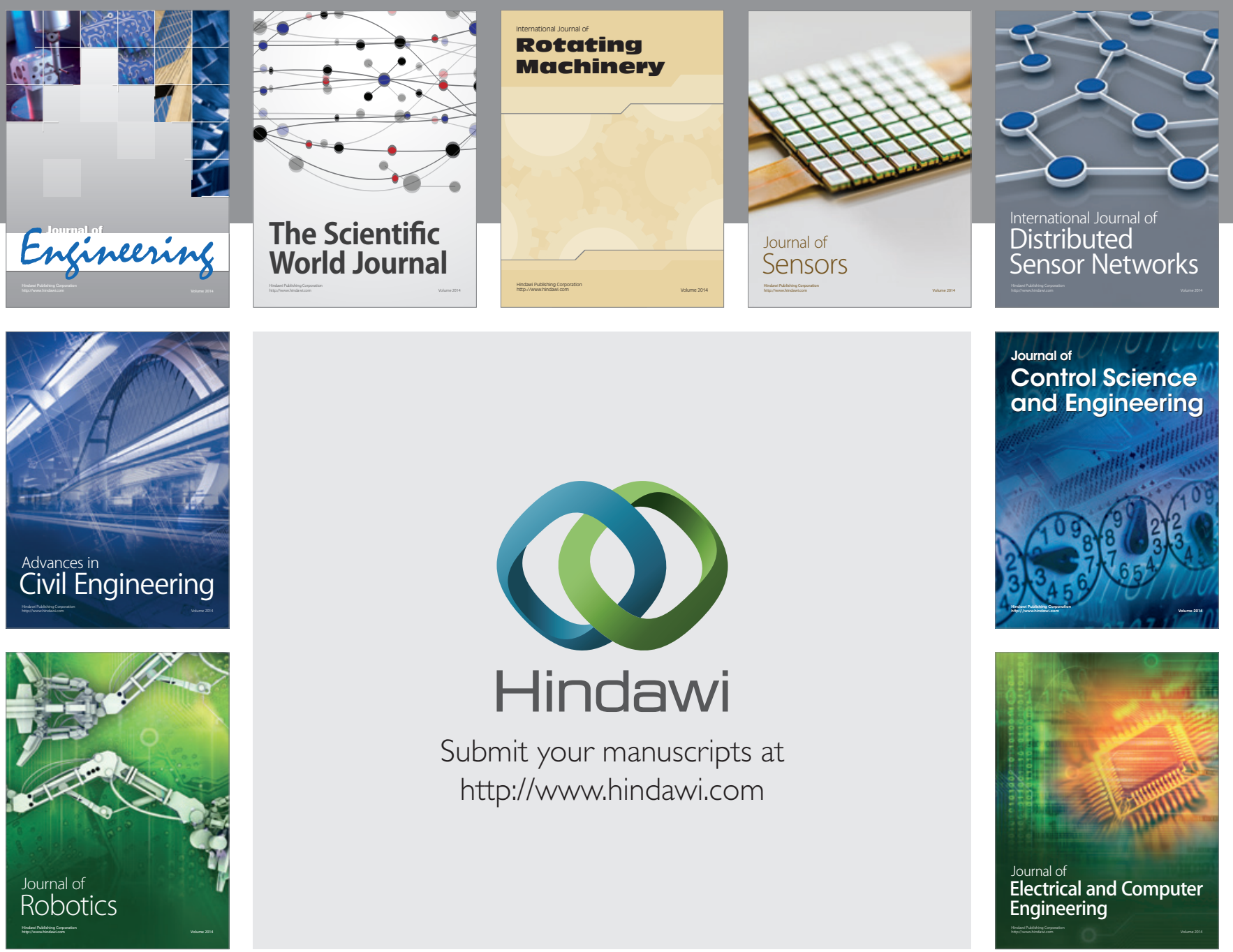

Submit your manuscripts at

http://www.hindawi.com
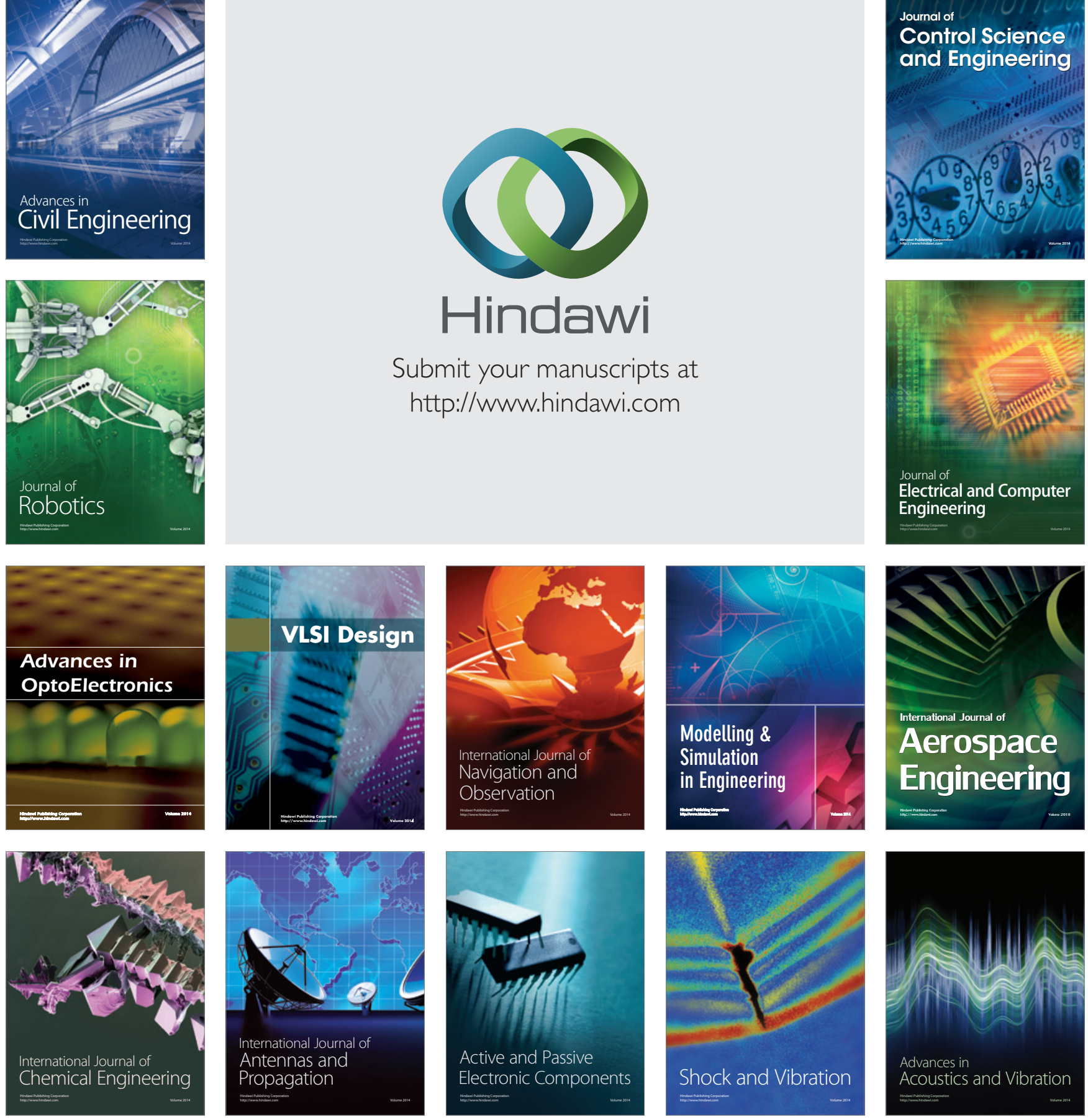